\title{
ON THE ORDER OF THE DIFFERENCE OF TWO MEROMORPHIC FUNCTIONS
}

\author{
S. M. SHAH
}

1. Introduction. In a recent paper [5] S. K. Singh has proved the following theorem:

Theorem A. Let

$$
f(z)=P_{1}(z) \exp \left(Q_{1}(z)\right)-P_{2}(z) \exp \left(Q_{2}(z)\right) \neq 0
$$

where $Q_{1}(z)$ and $Q_{2}(z)$ are polynomials of degree $\rho$ ( $\rho$ being an integer) and $P_{1}(z), P_{2}(z)$ are canonical products of order $<\rho$; then $\log M(r, f)$ $>A r^{p}$ where $A$ is some suitable constant; $M(r, f)$ being $\max |f(z)|$ for $|z|=r$.

In this note we prove the following extension of Theorem A.

Theorem 1. Let $P_{i}(z), i=1,2,3,4$ be canonical products or polynomials (not having any zero at origin) or constants (neither zero nor infinity) such that $P_{1}$ and $P_{3}$ have no common zeros, $P_{2}$ and $P_{4}$ have no common zeros, and let $\rho$ be a positive integer and

$$
\limsup _{r \rightarrow \infty} T\left({ }_{i}, P_{i}\right) / r^{\rho}=a_{i}<\infty .
$$

Further let

$$
Q_{1}(z)=a z^{\rho}+c_{1} z^{\rho-1}+\cdots, \quad Q_{2}(z)=b z^{\rho}+d_{1} z^{\rho-1}+\cdots
$$

be two polynomials such that $\max (|a|,|b|) \neq 0, k_{1}, k_{2}$ two integers or zeros and

$$
\begin{aligned}
F(z) & =z^{k_{1}}\left\{P_{1}(z) / P_{3}(z)\right\} \exp \left(Q_{1}(z)\right)-z^{k_{2}}\left\{P_{2}(z) / P_{4}(z)\right\} \exp \left(Q_{2}(z)\right) \\
& \neq 0 .
\end{aligned}
$$

Write

$$
\begin{aligned}
& D(F)=\{\max (|a|,|b|,|a-b|)-\min (|a|,|b|,|a-b|\} / \pi, \\
& t(F)=\underset{r \rightarrow \infty}{\liminf T(r, F) / r^{\rho}, \quad J(F)}=\underset{r \rightarrow \infty}{\limsup } T(r, F) / r^{\rho}, \\
& B_{1}=\underset{r \rightarrow \infty}{\limsup } T\left(r, P_{1} / P_{3}\right) / r^{\rho}, \quad B_{2}=\underset{r \rightarrow \infty}{\limsup } T\left(r, P_{2} / P_{4}\right) / r^{\rho}, \\
& A_{1}=\underset{r \rightarrow \infty}{\limsup } T\left(r, P_{1} P_{4}\right) / r^{\rho}, \quad A_{2}=\underset{r \rightarrow \infty}{\limsup \sup } T\left(r, P_{2} P_{3}\right) / r^{\rho}, \\
& A_{3}=\limsup _{r \rightarrow \infty} T\left(r, P_{3} P_{4}\right) / r^{\rho} .
\end{aligned}
$$

Presented to the Society, February 27, 1960; received by the editors December 29, 1959 and, in revised form, March 14, 1960 and May 6, 1960. 
Then

$$
\begin{aligned}
& t(F) \geqq D(F)-\sum_{i=1}^{2} B_{i} \geqq D(F)-\sum_{i=1}^{4} a_{i} \\
& t(F) \geqq|a-b| / \pi-\min \left\{2 \sum_{i=1}^{2} A_{i}, 3 \sum_{i=1}^{2} B_{i}\right\}, \\
& t(F) \geqq \max \{|a|,|b|\} / \pi-2 \sum_{i=1}^{2} B_{i} .
\end{aligned}
$$

If further $\sum_{i=1}^{2} B_{i}=0$ and either $\min (|a|,|b|)=0$ or $\arg a=\arg b$ $+k \pi$ ( $k$ integer or zero) then

$$
t(F)=\Im(F)=\max \{|a|,|b|,|a-b|\} / \pi .
$$

We assume here that the right-hand expressions of (1.4)-(1.6) (and of (2.1), (2.3), (2.4) below) are positive, for otherwise these results are trivially true.

J. Clunie [3] proved that if $f(z)$ and $g(z)$ are two integral functions of orders $\rho_{1}$ and $\rho_{2}\left(<\rho_{1}\right)$, then $f^{\prime}(z) g(z)-f(z) g^{\prime}(z)$ is of order $\rho_{1}$ and raised the question about the order of this function when type of $g(z)$ be less than type of $f(z)$. We prove here

TheOREM 2. Let $f_{i}(z)(i=1,2)$ be meromorphic functions of order $\leqq \rho(\rho>0)$ and write

$$
\begin{array}{r}
\limsup _{r \rightarrow \infty} T\left(r, f_{i}\right) / r^{\rho}=\alpha_{i}, \\
\limsup _{r \rightarrow \infty}\left\{\bar{N}\left(r, 1 / f_{i}\right)+\bar{N}\left(r, f_{i}\right)\right\} / r^{\rho}=\gamma_{i}, \\
f(z)=f_{1}^{\prime}(z) f_{2}(z)-f_{1}(z) f_{2}^{\prime}(z) .
\end{array}
$$

Suppose $\alpha_{1}>0, \alpha_{2}<\infty$. Then (i) $f(z)$ is of order $\rho$, maximal type if $\alpha_{1}=\infty$ and mean type if $\alpha_{1}<\infty, \alpha_{2}=0$; (ii) $f(z)$ is of order $\rho$ mean type if $\alpha_{1}<\infty$ and either (ii.a)

$\alpha_{1}>\left(1+2 A k^{8 \rho}\right) \alpha_{2} ; \quad A=\frac{1}{\log k} \log \left(\frac{16 e k^{2}}{k-1}\right)+\frac{2(k+1)}{k-1}, \quad k>1 ;$

or

$$
\alpha_{1}>\alpha_{2}+\gamma_{1}+\gamma_{2}
$$

or

(ii.c) ${ }^{1}$

$$
3 \alpha_{2}<\alpha_{1}\left\{\theta\left(0, f_{1}\right)+\theta\left(\infty, f_{1}\right)-1\right\} .
$$

${ }^{1}$ For the definitions, see [6, Chapter 2]. 


\section{Proof of Theorem 1.}

LEMMA 1. Let $F(z)$ be given by (1.3), and $A$ be any number. Then

(2.1) $t(F) \geqq\{|A|-|A-a|-|A-b|\} / \pi-\sum_{i=1}^{2} B_{i}$

(2.2) $J(F) \leqq\{|A|+|A-a|+|A-b|\} / \pi+\sum_{i=1}^{2} B_{i}$.

Proof.

$$
\begin{aligned}
F(z) & =\exp \left(A z^{\rho}\right)\left\{z^{k_{1}} \exp \left((a-A) z^{\rho}+\cdots\right) P_{1} / P_{3}\right. \\
& \left.-z^{k_{2}} \exp \left((b-A) z^{\rho}+\cdots\right) P_{2} / P_{4}\right\} \\
& =\exp \left(A z^{\rho}\right) \phi(z) .
\end{aligned}
$$

Then

$$
\begin{aligned}
\left\{|A| r^{\rho}\right\} / \pi & \leqq T(r, F)+T(r, \phi)+O(1) \\
& \leqq T(r, F)+\left\{\frac{|a-A|+|b-A|}{\pi}+\sum_{i=1}^{2} B_{i}+o(1)\right\} r^{p}
\end{aligned}
$$

and (2.1) follows. Also

$$
J(F) \leqq|A| / \pi+\underset{r \rightarrow \infty}{\limsup } T(r, \phi) / r^{p}
$$

and (2.2) follows.

Lemma 2. Let $F(z)$ be given by (1.3). Then

$$
t(F) \geqq\{|a-b|-\min (|a|,|b|)\} / \pi-\sum_{i=1}^{2} B_{i} .
$$

Proof. We may suppose $|b| \leqq|a|$. Write $X=z^{k_{1}} \exp \left\{(a-b) z^{\rho}+\cdots\right\} P_{1} / P_{3} ; Y=z^{k_{2}} \exp \left(d_{1} z^{q^{-1}}+\cdots\right) P_{2} / P_{4}$. Then

$$
\begin{gathered}
F(z) \exp \left(-b z^{\rho}\right)=X-Y, \\
T(r, X) \leqq T(r, F)+\left\{|b| r^{\rho}\right\} / \pi+\left(B_{2}+o(1)\right) r^{p} .
\end{gathered}
$$

Further

$$
\begin{aligned}
\exp \left\{(a-b) z^{\rho}\right\} & =X z^{-k_{1}} P_{3} \exp \left(-c_{1} z^{\rho-1}+\cdots\right) / P_{1}, \\
\frac{|a-b| r^{\rho}}{\pi} & \leqq T(r, F)+\frac{|b| r^{\rho}}{\pi}+\left(B_{1}+B_{2}+o(1)\right) r^{\rho}
\end{aligned}
$$

and (2.3) follows. 
Lemma 3. Let $F(z)$ be given by (1.3). Then

$$
t(F) \geqq|| a|-| b|| / \pi-\sum_{i=1}^{2} B_{i} .
$$

We may suppose $|a|>|b|$. Write $F(z)=f_{1}-f_{2}$. Then

$$
\begin{aligned}
T\left(r, f_{1}\right) & \leqq T(r, F)+T\left(r, f_{2}\right)+O(1) \\
& \leqq T(r, F)+\left(\frac{|b|}{\pi}+B_{2}+o(1)\right) r^{r} .
\end{aligned}
$$

Further

$$
\begin{aligned}
\exp \left(a z^{\rho}+c_{1} z^{\rho-1}+\cdots\right) & =z^{-k_{1}} f_{1} P_{3} / P_{1}, \\
\left\{|a| r^{\rho}\right\} / \pi & \leqq\left\{T\left(r, f_{1}\right)+B_{1}+o(1)\right\} r^{\rho}
\end{aligned}
$$

and (2.4) follows.

Proof of (1.4). We may suppose $|a| \geqq|b|$. Then (1.4) follows from (2.1) (with $A=a$ ), (2.3) and (2.4).

PROOF OF (1.5). Write

$$
\begin{aligned}
& X=z^{k_{2}}\left\{P_{2} P_{3} / P_{4} P_{1}\right\} \exp \left\{(b-a) z^{\rho}+d_{1} z^{\rho-1}+\cdots\right\}, \\
& Y=z^{k_{1}} \exp \left(c_{1} z^{\rho-1}+\cdots\right), \\
& \quad \phi_{2}=(X-Y) P_{1} / P_{3} ; \quad \phi_{1}=\exp \left(a z^{\rho}\right) .
\end{aligned}
$$

Then $F(z)=-\phi_{1} \phi_{2}$. Let $\gamma_{i}$ be a common zero (if any) of $P_{3}$ and $P_{4}$ with multiplicity $k_{3, i}$ in $P_{3}$ and $k_{4, i}$ in $P_{4}$. Write

$$
\begin{array}{ll}
N_{1}(r)=\sum \min \left(k_{3, i}, k_{4, i}\right) \log r /\left|\gamma_{i}\right|, & k_{3, i} \neq k_{4, i} ;\left|\gamma_{i}\right| \leqq r, \\
N_{2}(r)=\sum k_{3, i} \log r /\left|\gamma_{i}\right|, & k_{3, i}=k_{4, i},\left|\gamma_{i}\right| \leqq r .
\end{array}
$$

Then it is easily seen that

$$
\begin{aligned}
& N(r, X)+N(r, 1 / X) \\
& \leqq N\left(r, 1 / P_{2} P_{3}\right)+N\left(r, 1 / P_{4} P_{1}\right)-N_{1}(r)-2 N_{2}(r)+O(\log r) \\
&(2.7) \quad \leqq \sum_{i=1}^{4} N\left(r, 1 / P_{i}\right)-N_{1}(r)-2 N_{2}(r)+O(\log r), \\
&(2.8) \quad N(r, 1 /(X-Y)) \leqq N\left(r, 1 / \phi_{2}\right)+N_{2}(r)+O(\log r)
\end{aligned}
$$

since only those zeros of $P_{3}$ can be zeros of $X-Y$ which are considered in $N_{2}(r)$. Further

(2.9) $N\left(r, \phi_{2}\right) \geqq \sum_{i=3}^{4} N\left(r, 1 / P_{i}\right)-N_{1}(r)-2 N_{2}(r)+O(\log r)$. 
To prove (1.5) we assume that the right-hand expression of (1.5) is positive. Hence either $|a-b|>\pi\left(A_{1}+A_{2}\right)$ or $|a-b|>\pi\left(B_{1}+B_{2}\right)$. In both cases we have by an extension of the second theorem of Nevanlinna [4, pp. 75-76]

(2.10) $T(r, X) \leqq N(r, X)+N(r, 1 / X)+N(r, 1 /(X-Y))+o\left(r^{p}\right)$. Now

$$
\begin{aligned}
T(r, F) & \geqq T\left(r, 1 / \phi_{1} \phi_{2}\right)+O(1) \\
& \geqq N\left(r, 1 / \phi_{2}\right)+O(1) \\
(2.11) \quad \geqq N(r, 1 /(X-Y)) & \geqq T(r, X)-N(r, X)-N(r, 1 / X)-N_{2}(r)+o\left(r^{p}\right) \\
& \geqq\left\{|a-b| r^{\rho}\right\} / \pi-2 T\left(r, P_{2} P_{3}\right)-2 T\left(r, P_{4} P_{1}\right)+o\left(r^{\rho}\right) .
\end{aligned}
$$

Also from (2.11)

$$
\begin{aligned}
T(r, F) \geqq & \left\{|a-b| r^{p}\right\} / \pi-T\left(r, P_{1} / P_{3}\right)-T\left(r, P_{2} / P_{4}\right) \\
& \quad-\sum_{i=1}^{4} N\left(r, 1 / P_{i}\right)+o\left(r^{p}\right) \\
\geqq & \left\{|a-b| r^{p}\right\} / \pi-3 T\left(r, P_{1} / P_{3}\right)-3 T\left(r, P_{2} / P_{4}\right)+o\left(r^{p}\right)
\end{aligned}
$$

and (1.5) follows.

Proof of (1.6). We may suppose $|a| \geqq|b|$.

Case (i): $|a-b| \leqq \pi\left(B_{1}+B_{2}\right)$.

Then from Lemma 1 we have with $A=a$,

$$
t(F) \geqq \frac{|a|-|a-b|}{\pi}-\sum_{i=1}^{2} B_{i} \geqq \frac{|a|}{\pi}-2 \sum_{i=1}^{2} B_{i} .
$$

Case (ii): $|a-b|>\pi\left(B_{1}+B_{2}\right)$. We have in the notation of the proof of (1.5)

$$
\begin{aligned}
T(r, F) \geqq & m\left(r, 1 / \phi_{1} \phi_{2}\right)+N\left(r, 1 / \phi_{2}\right)+O(1) \\
\geqq & \left\{|a| r^{\rho}\right\} / \pi-T\left(r, \phi_{2}\right)+N\left(r, \phi_{2}\right)+N\left(r, 1 / \phi_{2}\right)+O(1) \\
\geqq & \left\{|a| r^{\rho}\right\} / \pi-T\left(r, \phi_{2}\right)+N(r, 1 /(X-Y))-3 N_{2}(r) \\
& \quad+\sum_{i=3}^{4} N\left(r, 1 / P_{i}\right)-N_{1}(r)+O(\log r) \\
\geqq & \left\{|a| r^{\rho}\right\} / \pi-T\left(r, \phi_{2}\right)+T(r, X)-N(r, X)-N(r, 1 / X) \\
& \quad-3 N_{2}(r)-N_{1}(r)+\sum_{i=3}^{4} N\left(r, 1 / P_{i}\right)+o\left(r^{p}\right) \\
\geqq & \left\{|a| r^{p}\right\} / \pi-T\left(r, P_{1} / P_{3}\right)-\sum_{i=1}^{2} N\left(r, 1 / P_{i}\right)-N_{2}(r)+o\left(r^{p}\right) .
\end{aligned}
$$


Since $N_{2}(r) \leqq N\left(r, 1 / P_{4}\right)$, we have

$$
t(F) \geqq|a| / \pi-2\left(B_{1}+B_{2}\right) .
$$

Proof of (1.7). From (2.2) we have

$$
\begin{aligned}
J(F) \leqq & \min \{|a|+|a-b|,|b|+|a-b|,|a|+|b|\} / \pi \\
& +\sum_{i=1}^{2} B_{i} .
\end{aligned}
$$

From the hypothesis on $a$ and $b$ we have either
(i) $|a-b|=|a|+|b|$ or
(ii) $|a-b|=|| a|-| b||$.

In case (i) we have from (2.13), (1.5), (1.6), since $\sum_{i=1}^{2} B_{i}=0$,

$$
t(F) \geqq\{|a|+|b|\} / \pi \geqq J(F)
$$

and so (1.7) follows. In case (ii) we have from (2.13), (1.5), (1.6)

$$
t(F)=\Im(F)=\max \{|a|,|b|\} / \pi=\max \{|a|,|b|,|a-b|\} / \pi \text {. }
$$

3. Remarks on Theorem 1. If we write

$$
F(z)=\left(z^{k_{1}} \exp \left(Q_{1}\right) P_{1} P_{4}-z^{k_{2}} \exp \left(Q_{2}\right) P_{2} P_{3}\right) / P_{3} P_{4}
$$

we get

$$
\begin{aligned}
t(F) \geqq & \frac{1}{\pi} \max (|a|,|b|)-2 A_{1}-2 A_{2}-A_{3} \\
\Im(F) \leqq & \min \{|a|+|a-b|,|b|+|a-b|,|a|+|b|\} / \pi \\
& +\sum_{i=1}^{8} A_{i} .
\end{aligned}
$$

If $|a-b|>\pi \min \left(\sum_{i=1}^{2} B_{i}, \sum_{i=1}^{2} A_{i}\right)$, then we have from (2.12)

$$
t(F) \geqq \frac{|a|}{\pi}-\min \left\{2 a_{1}+a_{2}+2 a_{3}, 2 a_{1}+a_{2}+a_{3}+a_{4}\right\}
$$

and similarly

$$
t(F) \geqq \frac{|b|}{\pi}-\min \left\{a_{1}+2 a_{2}+2 a_{4}, a_{1}+2 a_{2}+a_{3}+a_{4}\right\} .
$$

The inequalities (3.1), (3.2) and (1.5) (with $\sum A_{i}$ ) give an interval $I(A)$ in which $t(F)$ and $J(F)$ lie; and (1.4), (1.5) (with $\left.\sum B_{i}\right),(1.6)$ and (2.13) give another such interval $I(B)$. It is possible to choose a function $F$ for which $I(A) \subset I(B)$ and another function $F$ for which $I(B) \subset I(A)$. 
EXAMPLE 1. Write

$$
\begin{aligned}
\alpha_{n} & =(-1)^{n+1}(n+1) \log (n+1), \quad \sum_{n=1}^{\infty} 1 / \alpha_{n}=\alpha, \\
P_{1}(z) & =P_{2}(z)=\prod_{n=1}^{\infty} E\left(z / \alpha_{n}, 1\right), \quad P_{3}(z)=P_{4}(z)=\prod_{n=1}^{\infty} E\left(z /\left(-\alpha_{n}\right), 1\right) .
\end{aligned}
$$

Let

$$
a>0, k>0, a k>8 \alpha, \quad F(z)=\left(c^{a z}-\epsilon^{-k a z}\right) P_{1} / P_{3} .
$$

By a known theorem $\left[1\right.$, p. 27] $P_{1} P_{3}$ is a function of zero type order 1. We have

$$
\begin{gathered}
A_{1}=A_{2}=0, \quad A_{3}=2 \alpha / \pi=B_{1}=B_{2} ; \\
I(A):\{a(1+k) / \pi, \quad(a(1+k)+2 \alpha) / \pi\}, \\
I(B):\{(a(1+k)-12 \alpha) / \pi,(a(1+k)+4 \alpha) / \pi\} .
\end{gathered}
$$

EXAmple 2. Let

$$
\left(\beta_{k}\right)_{1}^{\infty}
$$

be such that $\left|\beta_{1}\right|<\left|\alpha_{1}\right|<\left|\beta_{2}\right|<\left|\alpha_{2}\right|<\cdots, \alpha_{n} / \beta_{n}>0, \sum_{n=1}^{\infty} 1 / \beta_{n}$ $=\alpha$, where $\alpha_{n}, \alpha$ have been defined in Example 1. Let

$$
P_{3}^{*}(z)=\prod_{n=1}^{\infty} E\left(z / \beta_{n}, 1\right), \quad F(z)=\left(e^{a z}-e^{-k a z}\right) P_{1} / P_{3}^{*}
$$

where $a>0, k>0, a k>8 \alpha$ and $P_{1}$ is as in Example 1. Then

$$
B_{1}=B_{2}=0, \quad A_{1}=A_{2}=A_{3}=2 \alpha / \pi .
$$

Hence $t(F)=\Im(F)=a(1+k) / \pi$ as given by (1.7); whereas $I(A)$ is

$$
\{(a(1+k)-8 \alpha) / \pi,(a(1+k)+6 \alpha) / \pi\} \text {. }
$$

4. Proof of Theorem 2. (i) We have

$$
f=f_{2}^{2} \frac{d}{d z}\left(f_{1} / f_{2}\right)=f_{2}^{2}\left(f_{1} / f_{2}\right)^{\prime} .
$$

Further [2] if $k>1, r>r_{0}$, then ${ }^{2}$

$$
T\left(r, f_{1} / f_{2}\right)<A T\left(k^{3} r,\left(f_{1} / f_{2}\right)^{\prime}\right)+k_{1} \log r
$$

where $A=A(k)$ is defined in the statement of Theorem 2, and $k_{1}$ is a constant. Hence

2 The relation (4.1) follows from the inequalities (26), (5), (6) of [2]. 


$$
\begin{aligned}
T(r, f) & >T\left(r,\left(f_{1} / f_{2}\right)^{\prime}\right)-2 T\left(r, f_{2}\right)+O(1) \\
& >\frac{1}{A} T\left(r / k^{3}, f_{1} / f_{2}\right)-2 T\left(r, f_{2}\right)+O(\log r) \\
& >\frac{1}{A}\left\{T\left(r / k^{3}, f_{1}\right)-T\left(r / k^{3}, f_{2}\right)\right\}-2 T\left(r, f_{2}\right)+O(\log r) .
\end{aligned}
$$

Also for $r>r_{0}(\epsilon)$

$$
\begin{aligned}
T(r, f) & <2 T\left(r, f_{2}\right)+T\left(r,\left(f_{1} / f_{2}\right)^{\prime}\right)+O(1) \\
& <2 T\left(r, f_{2}\right)+(2+\epsilon) T\left(r, f_{1} / f_{2}\right)+O(1) \\
& <(2+\epsilon) T\left(r, f_{1}\right)+(4+\epsilon) T\left(r, f_{2}\right)+O(1) .
\end{aligned}
$$

Hence (i) follows. Further from (4.2) we have, if $\alpha_{1}<\infty$, for a sequence of values of $r \uparrow \infty$,

$$
T(r, f)>\frac{1}{A}\left\{\left(\alpha_{1}-\alpha_{2}\right)\left(r / k^{3}\right)^{\rho}\right\}-2 \alpha_{2} r^{\rho}-o\left(r^{\rho}\right) ;
$$

and so

$$
J(F) \geqq\left\{\alpha_{1}-\alpha_{2}\left(1+2 A k^{8 \rho}\right)\right\} / A k^{3 \rho}>0 .
$$

From (4.3) we get

$$
J(f) \leqq 2 \alpha_{1}+4 \alpha_{2}
$$

and (ii.a) is proved.

(ii.b-ii.c) We have

$$
f=f_{1} f_{2}\left(f_{1}^{\prime} / f_{1}-f_{2}^{\prime} / f_{2}\right) \equiv f_{1} f_{2} \phi .
$$

Then

$$
\begin{aligned}
T\left(r, f_{1}\right)-T\left(r, f_{2}\right) & \leqq T\left(r, f_{1} f_{2}\right)+O(1) \\
& \leqq T(r, f)+T(r, \phi)+O(1) .
\end{aligned}
$$

Further

$$
\begin{aligned}
T(r, \phi) & \leqq T\left(r, f_{1}^{\prime} / f_{1}\right)+T\left(r, f_{2}^{\prime} / f_{2}\right)+O(1) \\
& =O(\log r)+N\left(r, f_{1}^{\prime} / f_{1}\right)+N\left(r, f_{2}^{\prime} / f_{2}\right) \\
& \leqq O(\log r)+\sum_{i=1}^{2}\left\{\bar{N}\left(r, 1 / f_{i}\right)+\bar{N}\left(r, f_{i}\right)\right\}
\end{aligned}
$$

Hence 


$$
\begin{aligned}
T(r, f) \geqq & T\left(r, f_{1}\right)-T\left(r, f_{2}\right) \\
& -\sum_{i=1}^{2}\left\{\bar{N}\left(r, 1 / f_{i}\right)+\bar{N}\left(r, f_{i}\right)\right\}+O(\log r)
\end{aligned}
$$

and so

$$
J(f) \geqq \alpha_{1}-\alpha_{2}-\gamma_{1}-\gamma_{2}>0 .
$$

Further from (4.5) we have for $r>r_{0}(\epsilon)$,

$$
\begin{aligned}
T(r, f) & \geqq\left\{\theta\left(0, f_{1}\right)+\theta\left(\infty, f_{1}\right)-1\right\} T\left(r, f_{1}\right)-3 \alpha_{2} r^{p}-o\left(r^{p}\right), \\
J(f) & \geqq\left\{\theta\left(0, f_{1}\right)+\theta\left(\infty, f_{1}\right)-1\right\} \alpha_{1}-3 \alpha_{2}>0 .
\end{aligned}
$$

Added in proof. (i) The author wishes to thank the referee for his comments. (ii) Write $|a|=\alpha,|b|=\beta,|a-b|=\gamma$. If we suppose that $\alpha^{2} \leqq \beta^{2}+\gamma^{2}+\beta \gamma, \beta^{2} \leqq \gamma^{2}+\alpha^{2}+\gamma \alpha, \gamma^{2} \leqq \beta^{2}+\alpha^{2}+\beta \alpha$, then we can get, from (2.2), a sharper inequality for $\Im(F)$, than (2.13) or (3.2). In fact we can show, in this case, that

$\Im(F) \leqq \frac{1}{\pi}\left\{\frac{\sum \alpha^{2}+\left(6 \sum \alpha^{2} \beta^{2}-3 \sum \alpha^{4}\right)^{1 / 2}}{2}\right\}^{1 / 2}+\min \left(\sum_{1}^{2} B_{i}, \sum_{1}^{3} A_{i}\right)$

where $\sum \alpha^{r}=\alpha^{r}+\beta^{r}+\gamma^{r}$.

\section{REFERENCES}

1. R. P. Boas, Jr., Entire functions, New York, Academic Press, 1954.

2. Chi-Tai Chuang, Sur la comparison de la croissance d'une fonction Meromorphe et de celle de sa dérivée, Bull. Sci. Math. vol. 75 (1951) pp. 171-190.

3. J. Clunie, The derivative of a meromorphic function, Proc. Amer. Math. Soc. vol. 7 (1956) pp. 227-229.

4. R. Nevanlinna, Le therème de Picard-Borel et la thérie des fonctions meromorphes, Paris, Gauthier-Villars, 1929.

5. S. K. Singh, $A$ note on entire and meromorphic functions, Proc. Amer. Math. Soc. vol. 9 (1958) pp. 6-10.

6. H. Wittich, Neuere Untersuchungen über eindeutige analytische Funktionen, Berlin, Springer, 1955.

NORTHWESTERN UNIVERSITY, AND

Muslim University, Aligarh, India 